\title{
Gradhiva
}

GRADHIV

Revue d'anthropologie et d'histoire des arts

3 | 2006

Du Far West au Louvre : le musée indien de George

Catlin

\section{Compte rendu des Indiens chippewas qui ont voyagé parmi les Blancs}

\section{Maungwudaus}

Traducteur : Claudie Voisenat

\section{Q OpenEdition}

\section{Journals}

Édition électronique

URL : http://journals.openedition.org/gradhiva/246

DOI : 10.4000/gradhiva.246

ISSN : 1760-849X

\section{Éditeur}

Musée du quai Branly Jacques Chirac

\section{Édition imprimée}

Date de publication : 15 juin 2006

ISBN : 2-915133-26-3

ISSN : 0764-8928

\section{Référence électronique}

Maungwudaus, "Compte rendu des Indiens chippewas qui ont voyagé parmi les Blancs », Gradhiva

[En ligne], 3 | 2006, mis en ligne le 10 juin 2009, consulté le 15 septembre 2020. URL : http:// journals.openedition.org/gradhiva/246

Ce document a été généré automatiquement le 15 septembre 2020.

(c) musée du quai Branly 


\title{
Compte rendu des Indiens chippewas qui ont voyagé parmi les Blancs
}

\author{
Maungwudaus
}

Traduction : Claudie Voisenat

\section{NOTE DE L'ÉDITEUR}

La troupe des Iowas menée par Nuage-Blanc (White Cloud), avec $\mathrm{M}^{\mathrm{r}}$ Melody pour imprésario, quitta Paris au début de l'été 1845, nous connaissons exactement son lieu d'origine - Saint-Joseph, Missouri - et beaucoup d'éléments sur son départ et son retour auprès de la mission presbytérienne animée par le révérend Irvin, traducteur, imprimeur et " protecteur » d'un tout petit groupe de survivants de cette tribu dont la mémoire actuelle est cristallisée autour des tableaux que Catlin fit de leurs ancêtres. Les archives laissées par le missionnaire sont riches mais n'ont pas été encore retrouvées sauf le journal tenu tout au long du périple européen par l'un des Indiens alphabétisés.

La troupe d'Ojibwas (la seconde visible au Musée Catlin) qui arrive salle Valentino au mois d'août 1845 est autonome ; son leader est, en même temps, son impresario. Il s'agit de Maun-gwa-daus, «Le grand héros » ou « Le courageux », figure exceptionnelle qui mériterait une biographie. Né vers 1807 sur le rivage nord-ouest du lac Ontario dans un groupe mississauga, il entre très jeune dans l'école d'une mission méthodiste où il est baptisé sous le nom de George Henry. Il se dit parent de Peter Jones, autre Ojibwa converti, traducteur des Évangiles, auteur d'une autobiographie, d'une description des coutumes ojibwas et, surtout, voyageur en Europe. Devenu missionnaire lui-même, George Henry rédige, à partir de 1837, des Letters pour le Christian Guardian, journal méthodiste, où il vante les bienfaits de la conversion sur les mœurs des Indiens. Pour des raisons inconnues, il quitte son ministère en 1840 . Il devient interprète pour le gouvernement jusqu'en 1843 et, une fois sa charge résiliée, décide de monter une 
troupe indienne (a wild Indian troupe) après avoir rencontré des Ojibwas rentrés d'Europe, peut-être les premiers à s'être produits, en 1844, dans le Musée Catlin. Reprenant son nom indien, Maun-gwa-daus fait une tournée aux États-Unis en 1844, s'embarque à New York en mars 1845, passe trois mois en Angleterre, avant d'arriver à Paris. Sa femme accouche de leur fils salle Valentino, dans le Musée indien. Reçue par Louis-Philippe, la troupe donnera des spectacles en extérieur, à Saint-Cloud, puis, sous la conduite protectrice de Catlin, se rendra en Belgique où, décimée par les maladies, elle décide de repartir. Man-gwa-daus continuera ses spectacles outre-Atlantique avant de reprendre sa vie indienne tout en « jouant ", jusqu'à la fin de ses jours, le rôle du visiteur des Blancs. Nous donnons ici la traduction de son journal, publié à ses frais à Boston, en 1848, en marge de ses spectacles: An Account of the Chippewa Indians who have been travelling among the Whites. Notons enfin que Maun-gwa-daus a été plusieurs fois dessiné, peint et photographié. Nous possédons une petite dizaine d'images de Catlin où il apparaît, très jeune d'allure, un daguerréotype de 1851 le représente avec tous ses attributs de chef, ainsi qu'un (ou deux) autre cliché de la troupe. La mise aux enchères, en décembre 2002, du portrait de Maun-gwa-daus par Paul Kane - sans doute réalisé en 1851 à Toronto - a suscité une véritable émotion patrimoniale au Canada, où l'État a été requis de l'acquérir'.

DF.

1 Relation du voyage des Indiens chippewas parmi les Blancs aux États-Unis, en Angleterre, Irlande, Écosse, France et Belgique; avec de très intéressants épisodes sur les caractéristiques générales des Anglais, Irlandais, Écossais, Français et Américains concernant leur hospitalité, particularités, etc., écrit par Maungwudaus, Indien autodidacte de la nation chippewa, pour son plus jeune fils, Noodinokay, dont la mère est morte en Angleterre.

2 Je ne demanderai pas son indulgence au lecteur. La courte notice qui me présente à la fin de ce compte rendu l'incitera à excuser mon usage maladroit de la langue anglaise.

Nous avons quitté les rives du lac Huron en 1843, visité les villes de Détroit, Cleveland, Buffalo, Utica, Albany, New York et d'autres encore. Avons quitté New York pour l'Angleterre le premier jour de mars avec le bateau appelé Victoria, accosté en Angleterre, à Portsmouth, le 26 du même mois. Pendant la traversée, notre mât du milieu a été emporté par le vent. Les vagues étaient comme des montagnes, nous n'avons pas eu le mal de mer, seulement été un peu blessés parfois quand nous étions jetés hors de nos couchettes. Quelquefois les vagues entraient dans notre cabine et nous forçaient à prendre un bon bain d'eau de mer. Les tonneaux de farine et de grains se sont détachés et se sont heurtés les uns aux autres; répandant tout leur contenu. Les rats ont fait un grand festin. Après qu'ils eurent rempli leurs ventres, toutes les nuits ont été pénibles; ils aidaient les vagues à nous tourmenter, nous mordant les orteils ou le nez. La mer pendant la nuit était comme la brûlure du feu. 


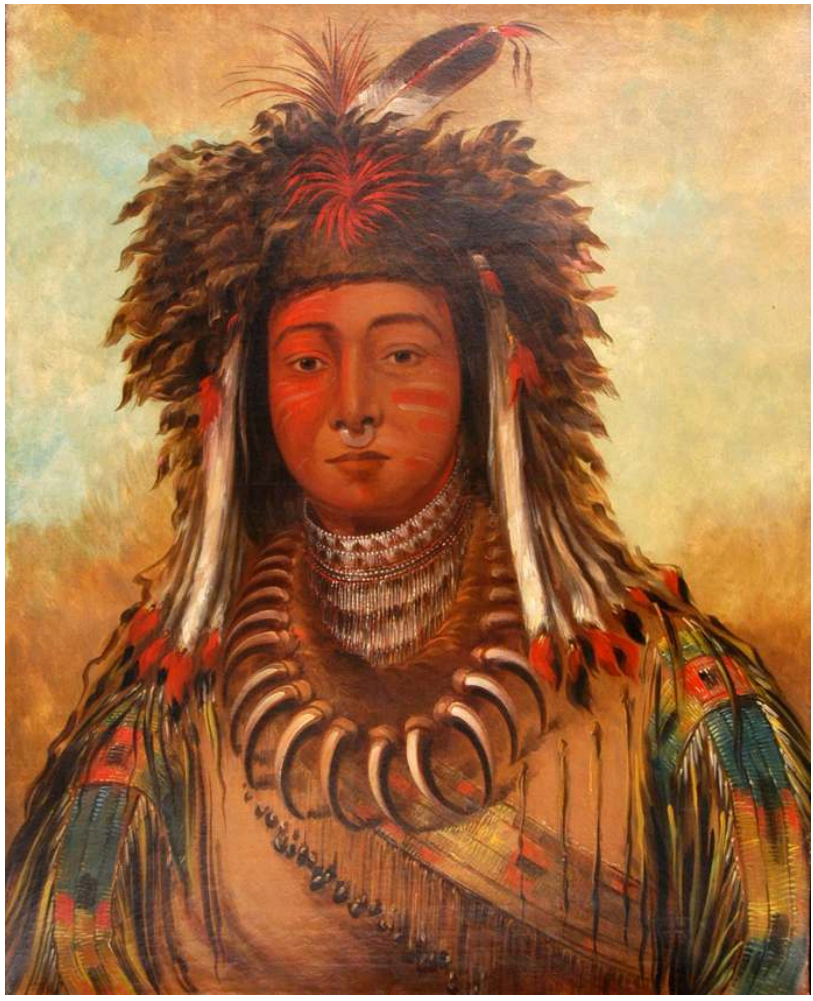

(c) musée du quai Branly

4 Nous sommes arrivés à Portsmouth le 26 du même mois. Portsmouth est un endroit immense pour les bateaux. Nous sommes allés voir le bateau de guerre de Lord Nelson et nous avons vu l'endroit où il est tombé quand il a été tué. Les officiers qui vivaient sur le bâtiment ont été très aimables avec nous. Le grand chef de guerre sur la mer nous a emmenés sur le chantier naval où ils construisent beaucoup de bateaux de guerre. Un autre chef de guerre nous a invités et nous a montré tous les guerriers qui sont sous ses ordres à la caserne.

5 De Portsmouth nous sommes allés à Londres, et sommes restés longtemps dans cette ville merveilleuse, nous produisant tous les jours à l'Egyptian Hall à Piccadilly. Cette ville fait environ dix miles de large mais en certains endroits elle est longue de vingt miles.

6 Les gens de cette ville sont comme les moustiques en Amérique pendant la saison d'été, aussi nombreux et se piquant les uns les autres pour assurer leur subsistance. Beaucoup sont très riches et beaucoup très pauvres; environ 900 naissances et 1100 morts chaque semaine dans cette seule ville. Il y a beaucoup de ponts, de pierre ou de fer, audessus de la Tamise. Les bateaux à vapeur ne sont pas aussi beaux que ceux d'Amérique. La plupart des maisons ont une couleur plutôt sombre parce qu'il y a trop de fumée.

7 Beaucoup de dames et de messieurs se promènent en carrosse. Les carrosses, les serviteurs et les chevaux sont couverts d'or et d'argent. Ils sont des centaines à marcher dans les parcs, pendant que leurs serviteurs « derrière eux » font prendre l'air à de petits chiens. Les femmes anglaises ne peuvent pas se promener seules, elles doivent toujours être aidées par des hommes. Et elles font porter leurs bébés par leurs maris pendant qu'elles se promènent. 
8 M. Harris nous a emmenés dans la maison de la reine. C'est une femme petite mais belle. Il y a beaucoup de femmes plus belles qu'elle. Le prince Albert est un bel homme bien bâti. La maison de la reine est grande. Nous avons été fatigués avant d'avoir visité toutes les pièces. De nombreux guerriers avec leurs épées et leurs fusils se tiennent à l'extérieur guettant l'ennemi. On nous a dit qu'elle a trois ou quatre maisons aussi grandes dans d'autres endroits. Celle que nous avons vue, disent-ils, est trop petite pour elle, et ils sont en train d'en construire une plus grande sur un des côtés.

Quand elle sort, elle a un grand nombre de guerriers devant et derrière, qui la gardent, la plupart ont sept pieds de haut. Leurs manteaux et leurs képis sont en acier, une longue crinière de cheval blanche ondule sur leur tête. Ils portent de hautes bottes, de longs gants et des culottes de daim blanc. Leurs épées, fusils et tout ce qu'ils ont, sont tenus très propres et brillants. Leurs chevaux sont tout noirs, avec beaucoup d'argent et d'or sur eux. Ils ne rasent pas la partie supérieure de leurs bouches mais se laissent pousser de longues moustaches [beards, les barbes] ce qui leur donnent un air féroce et sauvage comme nos chiens américains quand ils portent des écureuils noirs dans leurs gueules.

10 La noblesse, les ministres et la Société des Amis nous ont invités presque tous les jours à prendre le thé. Parfois nous passions près de deux heures à manger; les assiettes, couteaux, fourchettes, cuillers et tout ce qui était utilisé pour manger étaient en or et en argent. Les têtes des serviteurs étaient poudrées de blanc; ils nous ont donné beaucoup de beaux présents, et nous ont permis de voir beaucoup de choses que d'autres n'ont jamais vues. Nous sommes allés entendre Lord John Russell et Sir R. Peel parler au Conseil ; sommes aussi allés à la lower house et avons écouté les orateurs. Nous avons aimablement été invités à dîner avec Daniel o'Connell. Il a été très aimable avec nous. Nous avons traversé le tunnel sous le lit de la Tamise. Les bateaux naviguaient audessus de notre tête pendant que nous marchions au-dessous.

11 Notre chef de guerre a tiré un daim dans le parc, droit au cœur à trois cents yards et il est tombé mort devant quatre mille dames et messieurs. C'était fait pour les amuser. Voyageant en chemin de fer (Great Western Railway), la locomotive a abattu plusieurs freux ou corbeaux qui volaient au-dessus de la voie ferrée. Au Jardin zoologique, nous avons vu trois hommes partir pour le pays des étoiles. Ils avaient quelque chose de très grand et de la forme d'une vessie au-dessus de leur tête ; ils appelaient cela un ballon. Un homme nous dit: "Vous voyez maintenant que les Anglais peuvent aller voir le monde d'en haut avec leurs corps. » Lord Bloomfield nous a invités à voir les canons de Woolwich. Ils sont si gros que trois d'entre nous ont pu entrer sans peine à l'intérieur.

Ils disent qu'il y a huit mille épouses communes dans la ville de Londres. Ils disent qu'elles sont autorisées à marcher dans les rues chaque nuit pour la sécurité des femmes mariées. Les officiers anglais nous ont invités à manger avec eux à la caserne dans nos costumes indigènes. Quand le thé a été servi, les dames ont été amenées à la table comme des femmes malades. Nous avons passé près de deux heures à manger. Les dames étaient très bavardes. Elles sont très belles, leurs tailles, mains et pieds sont très petits, leurs cous sont plutôt plus longs que ceux de nos femmes. Elles portent la tête penchée de côté sur une épaule ; elles tiennent le couteau et la fourchette avec les deux premiers doigts et le pouce de chaque main; les deux derniers ne leur servent à rien, mais dépassent sur le côté, comme nos harpons à poisson.

13 Les officiers anglais ont une belle apparence, noble et digne. Leur voix, quand elle sort de leur bouche, ressemble à celle d'un crapaud buffle. Leur seul défaut est de faire trop 
de cérémonies inutiles en mangeant, comme, permettez-moi Sir, ou $\mathrm{M}^{\mathrm{rs}}$ de mettre ceci dans votre assiette. Si vous le souhaitez Sir, merci, vous êtes très aimable Sir ou $\mathrm{M}^{\mathrm{rs}}$ puis-je avoir le plaisir de vous aider?

Beaucoup d'Anglais ont de très gros estomacs à cause de toute la bière qu'ils boivent. Ceux qui boivent du vin ou du brandy ont le nez qui ressemble à des fraises mûres.

Lorsque nous avons été prêts à partir, un des officiers nous dit: nos dames seraient heureuses de vous serrer la main, et nous leur avons serré la main. Alors ils ont parlé entre eux et un autre officier nous a dit «Amis, nos dames pensent que vous ne leur avez pas assez présenté vos respects, elles souhaitent que vous les embrassiez »; alors nous les avons embrassées selon nos coutumes sur chaque joue. «Mais! Ils nous ont embrassées sur les joues, en voilà une curieuse façon d'embrasser.» Alors un autre officier nous dit, «Messieurs, nos jolies squaws ne sont pas encore satisfaites, elles veulent être embrassées sur la bouche.» Alors nous les avons embrassées sur la bouche; et il y eut un grand cri parmi les chefs de guerre anglais. Say-say-gon, notre chef de guerre dit alors aux dames dans notre langue : «C'est tout ce à quoi vous êtes bonnes; parce que comme femmes vous n'êtes bonnes à rien.» Les dames voulurent que je leur traduise ce que le chef leur avait dit. Je leur expliquai qu'il avait dit qu'il espérait que les officiers l'inviteraient très souvent, pour qu'il puisse embrasser à nouveau les belles dames. Alors elles dirent: "C'est vrai? alors nous dirons à nos hommes de vous inviter à nouveau, car nous aimons être embrassées très souvent, dites-le lui. » Et elles mirent des anneaux d'or à nos doigts et des épingles d'or sur nos poitrines. Après les avoir remerciées pour leur gentillesse, nous sommes montés dans notre voiture et sommes rentrés dans nos appartements.

16 Le grand chef de guerre avec le gros nez, le Duc de Wellington, nous a invités chez lui et a été très aimable avec nous. Lui et son fils nous ont donné de beaux présents.

Sir Augustus d'Este, cousin de la Reine et fils du Duc de Sussex, nous a très souvent invités à prendre le thé avec lui. C'est un grand ami des Indiens, il nous a présenté beaucoup de ses amis. Ce grand homme est un invalide et n'est pas capable de marcher seul.

18 L'archevêque de la cathédrale de Canterbury a été très agréable avec nous; il nous a tout montré dans la cathédrale, les œuvres curieuses et merveilleuses des anciens Bretons. Il dit que cet édifice est vieux de treize cents ans. C'est le plus curieux, le plus grand et le plus beau que nous ayons vu. Nos flèches ne pourraient pas atteindre le haut de son clocher.

19 Nous sommes allés voir Douvres, nous sommes passés par des routes souterraines à travers la roche.

20 Nous sommes allés en France; sommes restés cinq mois à Paris avec les Curiosités Indiennes de Catlin. Avons serré la main de Louis-Phillippe (sic) et de toute sa famille dans le parc appelé Saint-Cloud. Nous leur avons donné une petite danse de guerre, avons tiré avec les arcs et les flèches sur une cible, joué au ballon ; nous avons aussi fait naviguer notre canoë d'écorce de bouleau sur le lac artificiel, au milieu des cygnes et des oies. Il y avait avec eux environ quatre mille personnes, dames et messieurs. Dans l'après-midi, nous avons dîné avec lui dans son palais. Il nous a beaucoup parlé de son voyage en Amérique quand il était un jeune homme. Il nous a donné douze médailles d'or et d'argent ; il nous a montré toutes les pièces de sa maison. 

Beaucoup de messieurs ne rasent jamais leur visage, on dirait qu'ils n'ont pas de bouche. D'autres portent la barbe seulement sur la partie supérieure de la bouche, et on dirait qu'ils ont des queues d'écureuils noirs qui pendent de chaque côté.

(a) Lafontaine, le grand hypnotiseur, nous a invité à son spectacle. Il nous a donné une aiguille pour piquer ceux qu'il fait dormir, et nous avons planté l'aiguille à travers leurs sourcils et dans la main entre le pouce et l'index, et ils ne se sont rendu compte de rien. Il a demandé à la jeune femme de mettre ses bras autour de nos cous et de nous embrasser en disant en même temps "mon cher». Nous avons laissé sur ses joues un peu de la peinture que nous avions au visage. Un autre Français a fait des moulages de nos têtes en plâtre de Paris; certains de nos jeunes gens ont été effrayés. Louis-Phillippe a demandé à $\mathrm{M}^{\mathrm{gr}} \mathrm{J}$. Gudin de peindre nos ressemblances sur une grande toile, et quand cela a été fait, il l'a prise dans son palais.

De Paris, nous sommes allés à Bruxelles. Le roi de ce pays a été très aimable avec nous. Les Belges sont comme les Français. Là les pauvres Aunimuckwuh-um et Mishimaung moururent de la petite vérole. Après avoir visité d'autres villes à la frontière de l'Allemagne, nous sommes retournés à nouveau à Londres; là notre très respecté chef de guerre Say-say-gon mourut de la même terrible maladie que les autres. Ces trois hommes n'avaient pas permis aux médecins blancs de les vacciner. Ils disaient que nous étions fous de nous en remettre aux soins des Blancs - nous-mêmes avons été sauvés par ce simple remède, grâce à la gentillesse de nos amis les Quakers. 
Affiche annonçant une conférence au Rumford Hall par le chef Maun-gwa-daus et d'autres Indiens chippewa, Boston, 1850.

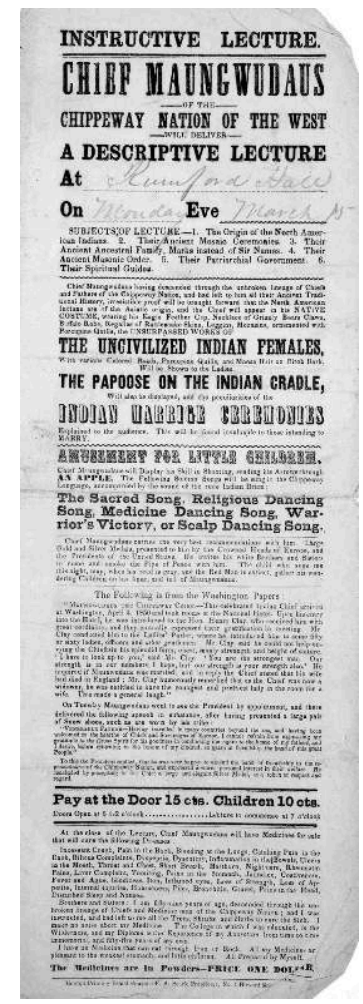

American Memory Collection, the Library of Congress.

Lorsque nous étions à Ipswich, nous avons rendu visite à $\mathrm{M}$. Thomas Clarkson. Il a été très heureux de nous voir bien qu'il ait été très malade. Sa charmante fille nous a donné un bon dîner et de beaux présents. Cet homme grand et bon est mort peu après que nous l'eûmes quitté.

À Norwich, M. J. J. Gurney, le grand homme des Quakers nous a invités dans sa maison appelée Earlham. Lui et sa femme, de Philadelphie, ainsi que son fils, nous ont donné de riches présents. Ce grand homme est mort d'une chute de cheval pendant notre séjour en écosse.

Tandis que nous étions à Norwich, nous avons vu tuer un homme; il a été exécuté parce qu'il avait tué une femme à Yarmouth pour un peu d'argent. Des milliers de personnes se sont rassemblées là où l'homme devait être tué, devant une grande maison de pierre, au sommet d'une colline, bien longtemps avant l'heure prévue pour l'exécution. Puis ils ont fait sortir l'homme qu'ils voulaient tuer. Ils l'ont fait se tenir sur une estrade avec une solide corde autour du cou, et quand celui qui enseigne la sagesse et la religion chrétienne eut prié pour lui, ils lui ont rabattu sa coiffe blanche sur le visage et ont attaché le bout de la corde qu'il portait au cou à une poutre au-dessus de sa tête. Alors un autre homme a fait tomber l'estrade sur laquelle se tenait le condamné ; celui-ci est brusquement descendu un peu, suspendu dans les airs par la corde qui était autour de son cou, les mains attachées dans le dos. Alors il a commencé à battre des pieds et à se tordre pour rester en vie et l'un des meurtriers est descendu en courant, s'est accroché à ses jambes et l'a tiré vers le bas, le tuant très vite. Ils ont dit qu'il n'était pas digne de vivre sur terre, mais qu'ils croyaient qu'il était allé dans la contrée heureuse de l'autre monde, où il ne pourra plus jamais faire de mal. 
31 Nous sommes passés par Sheffield, Manchester, et beaucoup d'autres villes entre les deux ; vu beaucoup de bonnes gens et de choses merveilleuses.

De Liverpool à Dublin, nous sommes allés voir le Père Matthew. Nous nous sommes produits à la Rotonde, ainsi que dans le Jardin zoologique; chaque soir trois mille personnes venaient nous voir; sommes allés vers le sud puis à nouveau vers le nord, Belfast et Londonderry. Les Irlandais ont beaucoup de cœur. Les paysans font du feu avec de la tourbe, un grand nombre d'entre eux sont très pauvres; le gouvernement britannique les commande.

33 Nous avons fait la traversée vers l'écosse et sommes arrivés en un lieu appelé Androsson; nous sommes allés voir la maison de R. Burns, petite avec un toit de chaume. Nous sommes allés voir le chêne de Wallace, près de Paisely ; sommes allés à Glasgow et édimbourg. édimbourg est remplie d'écossais; la ville nouvelle est très belle, mais la vieille ville est plutôt dégoûtante. Toutes les ordures sont jetées dans les rues et des charrettes viennent les emporter avant que les gens ne se lèvent, malgré tout l'odeur reste insupportable toute la journée. Un des chefs nous a raconté que quelques années auparavant, un écossais qui était né dans la ville mais s'en était éloigné pendant plusieurs années avait dit en y revenant : «Rien ne vaut son chez soi »; puis comme il commençait à sentir l'odeur qui montait des rues, il dit : « Ah! cher vieil édimbourg, je te retrouve enfin ». Les chefs écossais nous ont montré la couronne d'écosse dans le Château, ainsi que le Palais. Nous sommes allés voir environ soixante-dix jeunes gens qui vont devenir des hommes-médecine. Ils avaient trente cadavres et étaient en train de les dépecer et de les couper comme nous le faisons de la venaison.

Les écossais sont très religieux et travailleurs, ils montrent beaucoup de cœur envers les étrangers. Ils respectent très strictement le dimanche. Un grand nombre d'entre eux ne boivent jamais d'alcool ; leur pays est montagneux. Les vieux, hommes et femmes, adorent le tabac à priser. Ils le portent dans des cornes de bélier et ils s'en mettent une cuillerée dans chacune des deux narines; de ce fait leurs mots ont une résonance nasale, comme le grognement d'un cochon.

À Glasgow, deux de mes enfants sont morts, et un autre encore à Édimbourg ; les avons enterrés dans le cimetière de nos amis les Quakers. Après avoir visité d'autres villes au nord et au sud, nous sommes retournés en Angleterre. Ma femme est morte à Newark. Le vicaire a été très bon pour nous et nous a permis d'enterrer ses restes près de l'église.

36 Un jour que nous faisions un tour en ville dans nos costumes indigènes, nous avons vu un singe qui dansait dans la rue sur une boîte à musique, entouré d'une cinquantaine de jeunes gens qui le regardaient. Il était habillé comme un homme. Quand les jeunes gens nous virent, ils commencèrent à se moquer de nous, utilisant un langage très insultant et faisant grand bruit. En nous voyant, le singe oublia son spectacle, et comme nous le regardions, il enleva son képi rouge et nous fit un salut. Un gentleman qui était là dit au public : «Regardez le singe enlever son képi et saluer les étrangers. Qui les étrangers vont-ils juger les plus civilisés, vous ou le singe? Vous devriez avoir honte. Vous vous considérez peut-être meilleurs et plus sages que ces étrangers, mais vous vous trompez grandement. La façon dont vous les traitez leur montre que vous ne l'êtes pas, et vous êtes si stupides et ignorants que vous ne savez rien de tout cela. J'ai voyagé cinq ans parmi ces hommes, dans leur propre pays, et jamais, pas une fois, je n'ai été insulté, mais j'ai toujours été aimablement traité et respecté par chacun d'entre eux. Leurs petits-enfants ont de bien meilleures manières que les vôtres. Jeunes gens, le singe vous 
en donne pour les penny que vous lui avez jetés, il est digne de devenir votre professeur. » Nous avons alors lancé de l'argent au singe, qui est descendu de son estrade, a attrapé les pièces, a sauté de nouveau sur la boîte et a mis l'argent dans la bouche de son maitre. Comme il nous saluait à nouveau tandis que nous nous éloignions, nous avons entendu un des jeunes gens dire à ses amis: "Regardez le professeur faire un autre salut aux Indiens.» "Oui » dit un autre, "c'est pour t'apprendre puisque c'est toi qui t'es moqué des Indiens ».

37 Nous avons visité New Castle upon Tyne, Leeds, York, Birmingham et beaucoup d'autres villes; avons visité la maison de Shakespeare et sa tombe à Stratford on Avon. Nous avons visité la maison de Lord Byron. Le colonel Wildman a été très aimable avec nous ; allés à Nottingham et Londres à nouveau.

Nous avons quitté Londres le 23 avril 1848, dans un bateau appelé Yorktown, de New York. Le capitaine Seba a été très gentil avec nous pendant tout le voyage. Seize enfants allemands sont morts pendant le trajet, ainsi qu'une dame anglaise. Nous-mêmes n'avons pas eu le mal de mer. Les vagues étaient comme des montagnes; vu sept baleines et beaucoup de marsouins. Arrivés à New York le 4 juin, nous avons été très reconnaissants envers le Grand Esprit qui nous avait ramenés en Amérique.

Pendant le voyage, le capitaine Seba a fait très attention que personne ne fume des pipes ou des cigares dans le bateau ni ne boive de l'eau de feu, il interdisait les disputes et les bagarres. Mais après deux ou trois jours de mauvaise mer, les Anglais et les Allemands se sont un peu battus, parce qu'il n'y avait pas assez de place pour toutes leurs théières dans la cuisine. Personne n'a été sérieusement blessé, seuls quelques visages ont été un peu coupés et griffés, mais le chef en second leur a ensuite donné quatre ou cinq coups de corde sur le dos.

Le capitaine a autorisé le ministre du culte à prêcher dans sa cabine tous les dimanches. L'intérieur du bateau était nettoyé régulièrement, l'extérieur l'était tous les jours pour que les maladies ne puissent pas se développer. Il était comme un père pour nous ; tous les marins l'aimaient et lui obéissaient de bonne grâce.

41 Quand on pouvait voir le soleil, il le regardait avec une petite lunette, curieusement construite en cuivre jaune [laiton]; ainsi il pouvait nous dire où nous étions et à quelle distance de l'Angleterre et de l'Amérique. Parfois, il demandait à l'un de ses hommes de laisser filer dans l'eau un morceau de plomb attaché à une grande corde dont le bout était recouvert de suif. Quand on la remontait, il y avait du sable sur le suif, et lui, en le regardant et en examinant sa couleur, savait exactement où nous étions, il tirait une grande sagesse de l'observation du soleil et du sable.

Il avait aussi quelque chose dans un tube de verre qui ressemblait à du plomb fondu et, en le regardant, il pouvait toujours dire quand la tempête arrivait et préparer le bateau à l'affronter. Il était aussi bon qu'il était un grand homme.

43 À Ipswich, nous avons dîné avec nos amis les Quakers, ils étaient environ soixante; ils s'appelaient Alexanders et Ransoms. Après avoir mangé beaucoup de bonnes choses et quand les assiettes eurent été enlevées, on mit sur la table un fromage rond, petit mais haut, et l'un de nos amis les plus âgés nous dit : «Amis, voici notre fromage anglais; les plus pauvres d'entre nous ne peuvent pas se permettre d'en manger. Nous considérons qu'un repas n'est pas terminé tant que nous n'en avons pas mangé un peu, voulez-vous y goûter? Je dis oui. Voulez-vous y goûter, etc., jusqu'à ce que chacun d'entre nous en ait un morceau devant lui, et nous en mangeâmes beaucoup, parce qu'il venait de nos 
amis. Quand nous eûmes fini de manger, un docteur, dont le nom est F.W. Johnson, plaça sur la table ce qu'il appelle un microscope qui avait trois jambes de cuivre jaune et une petite lunette; puis il posa un très petit morceau du fromage que nous avions mangé sur une assiette propre et nous dit de le regarder à travers la petite lunette qui était sur les trois jambes de cuivre, et nous avons vu des centaines de vers y grouiller. Cela fit rire nos amis et nous essayâmes de rire aussi, mais nous étions en même temps terrifiés de savoir que nous en avions avalé des milliers. Quand nos amis virent que nous étions terrifiés, l'homme-médecine versa une goutte d'eau de pluie dans un verre propre, et nous le fit regarder lui aussi dans la petite lunette, et nous vîmes des centaines de créatures vivantes y nager ; certaines comme des bêtes, d'autres comme des serpents, ou comme des poissons, certaines avaient des cornes et d'autres non, certaines avec des jambes et certaines sans ; certaines avaient des roues de chaque côté de leur corps et se déplaçaient avec comme des bateaux à vapeur, s'accrochant, se poursuivant, se battant, se tuant et se mangeant les unes les autres. Alors l'un des plus vieux de nos amis nous dit : "Vous voyez, mes amis, vous ne devez pas penser que vous avez mangé des vers pour la première fois. Nous avalons des milliers d'entre eux chaque jour, dans la nourriture ou dans l'eau. Ils flottent dans les airs et nous les inhalons quand nous respirons; des milliers d'entre eux flottent aussi dans nos veines. Le Grand Esprit qui nous a faits, nous et les autres êtres vivants, est merveilleux en puissance et en sagesse. Nous espérons sincèrement que vous l'aimerez toujours et que vous obéirez à ce qu'il vous dit dans vos cœurs. » Pendant deux ou trois jours nous avons attendu que les vers nous mordent. Parfois nous les cherchions, pensant qu'ils pouvaient avoir beaucoup grossi pendant qu'ils étaient dans nos corps, mais nous n'avons ni senti leur morsure ni vu aucun d'entre eux. Nous avons souvent pensé depuis que nos amis doivent être un peu comme les ours qui aiment manger des vers et des asticots.

Depuis le 4 juin, nous avons visité beaucoup de villes entre New York et Boston; sommes allés voir Plymouth Rock où nos ancêtres ont vu pour la première fois des hommes blancs. Nous avons vu la première pierre foulée par l'homme blanc, et sommes allés dans le Pilgrim Hall. Partout, les Américains ont été très gentils avec nous ; ils ne sont pas aussi bien en chair que les Anglais, mais ils sont très persévérants dans ce qu'ils entreprennent. Ils respectent plus leurs femmes que les Anglais. Ils mettent leurs pieds sur les tables, les chaises et les cheminées quand ils fument le cigare ou lisent les journaux. Ils ne sont pas aussi esclaves de leur civilisation que les Anglais ; ils aiment le confort, un peu comme nous, plaçant une jambe sur l'autre genou pendant que nous nous prélassons au soleil. La nature humaine préfère le vrai confort à l'artifice.

Les maires de toutes les cités et villes de ce pays ont été très aimables en nous permettant d'utiliser leurs hôtels de ville et leurs préfectures de comté.

Maungwudaus est le fils de Pishikay qui, avec d'autres chefs, vendit beaucoup de terres au gouvernement britannique, à l'ouest du Canada. Maungwudaus est autodidacte : il a appris à parler anglais, à lire et à écrire après son mariage. Il est né et a été élevé dans les étendues sauvages et n'a jamais vécu dans une maison mais dans un wigwam. Il restait souvent pendant des mois, été comme hiver, sans rien au-dessus de sa tête. C'est un homme d'une intelligence rare et d'un grand cœur ; le meilleur interprète que le gouvernement et les missionnaires aient jamais eu. Son père et son grand-père étaient de grands hommes. Le nom de son grand-père était Aindussing Aunuckwodt ; il est mort et a été enterré sur la Grande Rivière, environ deux miles au-dessus d'un village appelé Galt, dans le canton de Dumfress à l'ouest 
du Canada.

M. William Heating, superintendant des Affaires indiennes.

\section{NOTES}

1. On ajoutera au récit européen de Catlin et aux articles cités dans la présentation de ce dossier deux contributions importantes : Frank Little, dans « Early Recollections of the Indians about Gull Prairie », (Michigan Pioneer and Historical Collections, vol. 27, 1896 : 330-338), rapporte ses souvenirs de jeune homme qui rencontre et écoute, vers 1850, dans le bazar de Gull Corners, l'impressionnant Maun-gwa-daus parlant de Catlin; Donald B. Smith, dans « Maungwudaus Goes Abroad» (The Beaver, automne 1976 : 4-9) propose, pour sa part, un parcours biographique.

\section{AUTEURS}

MAUNGWUDAUS 